\title{
Research on complexity of transport networks
}

\author{
Youbing Zhu \\ Chongqing College of Electronic Engineering, Chongqing, 401331, China \\ Email:happylover618@126.com
}

\begin{abstract}
Keywords: Transport networks, complexity, user equilibrium, unscaled.
Abstract. Depth study of the internal operating rules and mechanisms for transport network system theoretically comprehensive system to fully grasp the transportation network in the micro and macro levels of different complexity, improve traffic network system design and management, it has become China's social and economic development the significant demand. Based on the theory of complex networks, integrated management science, computer simulation traffic assignment model and technology disciplines, such as the complexity of network traffic study aims to analyze the general characteristics of large-scale traffic network topology has revealed micro and macro levels the intrinsic link between exploration of complex transportation network operating rules and mechanisms, provide new perspectives and decision-making basis for future design and management of transport networks.
\end{abstract}

\section{Introduction}

Scale-free networks are an important aspect of it [1]. Scale-free network refers to the network of distribution of power law distribution, due to its lack of a description of the problem characteristic scale is called scale-free networks [2, 3]. It has a real network the most common two characteristics: growth and preferential attachment of the first characteristics indicate that scale-free networks can continue to expand the second feature is the ability to connect two nodes means that differences can increase with the expansion of the network while [4-7]. Great initially connect more nodes can be formed more connections [8].

Research on scale-free networks, the preparedness of the transport network has been coordinated attack and destruction, prevent traffic congestion, improved network reliability [8]. They are extremely important theoretical and practical significance of scale-free networks unexpected failure has amazing strong toughness, derived from this feature is essentially a non-homogeneous topology of these networks [9]. Link almost all those nodes the hubs compared to those with only a small amount of non-essential nodes of the link [10]. Thus they will not be removed have a major impact on the network topology. this increases our scientific understanding of the transport network, some random removal and transportation nodes will not cause paralysis of the entire transport network, but if we deliberately to attack and destroy the Hub transport, will quickly lead to paralysis of the entire network is currently abroad on complex networks have some research results, most still in the exploratory stage, there is no a very sound theory $[11,12]$.

Microscopic analysis traveler time and money considerations path based on choice behavior, study topological properties of the whole network, network design, network efficiency and other properties of individual behavior due to the macro. Complex Networks Follow microscopic interactions between individuals of the system leads to macroscopic phenomena, micro and macroscopic properties can be linked, it can predict the behavior of complex systems-rich whole, including the self-organization, thus becoming the study of complex transportation network powerful tools.

\section{Urban Traffic Network Construction}

Real world transport networks are extremely complex, urban public transport network is an example of the definition: the network node represents the final bus lines from the site, the connection between the final site from the edges represent so many of the points and. a network of lines constitute the basic framework of urban public transport network. Taking into account the specific organization 
and operation of public transportation systems, we can be attributed to the two cases to represent the organization OD bus lines between Fig. 1 (a) represents the $i$ to $j$ and return from $j$ to $i$ line, and in some cases, city bus lines also Fig. 1 (b) a situation in which start and end point of the bus lines are i, constitute the public transport network referred to in the "ring".

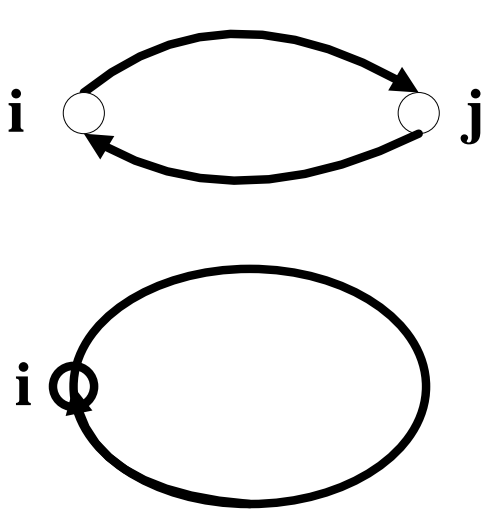

(a)

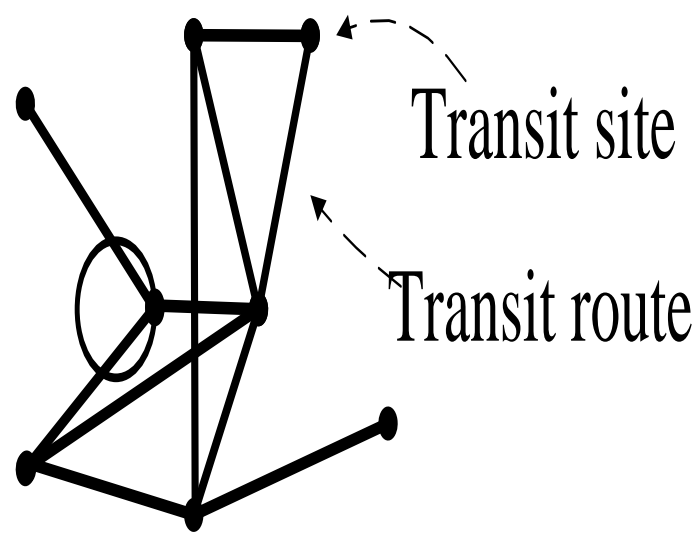

(b)

Figure 1. Simple example of urban public transport network

Based on the model, based on researchers by changing the factors put forward a variety of weighted network evolution model, trying to explain the formation mechanism more realistic actual network. This paper will elaborate on the content and status of basic mechanisms of evolution of network structure stability is also an important part of this part of the study, which studies complex networks and the ability to withstand unexpected failure malicious attacks, which can prevent emergencies and for people to improve the robustness of the system play a role in this part of the study is normally achieved by computer simulation.

\section{Topological statistical characteristic of complex networks}

With the deepening of globalization, accelerate the pace of social life, transportation supply chain network nodes involved more and more and more complex structure, facing more pressures and challenges, consumers increasingly transportation service quality requirements higher. The use of complex network theory can reveal the overall macroscopic properties, dynamics of transportation supply chain network formation change process, analyze the stability and resilience transportation service supply chain network, to help us with a new perspective to look at the transportation supply chain network. Degree and degree distribution point is defined two networks are very important for all statistical characteristics of nodes and edges of the total number of nodes connected to the specific formula is as follows:

$$
k_{i}=\sum_{j} a_{i j}
$$

Where aij is the network adjacency matrix A of the elements used to indicate the presence or absence of the edge between two points, aij $=1$ means there is an edge between the point $\mathrm{i}$ and the point j, otherwise not. In different networks represented the meaning is different in social networks, it can be used to indicate the degree of the individual's influence and importance, the greater the individual, the greater their influence, role in the organization of it, and vice versa . represents the probability distribution network in all points of the distribution function of the dead, in order to reduce the statistical error and improve the fitting precision of expression generally used is the cumulative probability distribution function:

$$
P\left(k>k^{\prime}\right)=\sum_{k=k^{\prime}}^{\infty} p_{k^{\prime}}
$$

Average path length is calculated as follows: 


$$
D=\frac{1}{N(N-1)} \sum_{i \neq j} d_{i j}
$$

In the empirical study, researchers have found many interesting phenomena, various transportation networks have a lot in common, they all obey the power-law degree distribution point, with or without small-scale nature of the world and nature. They also recognize that the traffic flow in a transportation network plays a very important role, to the point of strength, weight and other topological properties of in-depth analysis and research.

\section{Data analysis and results}

Space Network has especially transportation network studies obtained in a number of research areas GIS. GIS vast majority of applications only use traditional methods such as the shortest path search, the nearest building or commercial outlets search, compute nodes in the network or roads flow capacity. does not provide applications and software beyond the standard kit further than application functionality, geographers who are not familiar with such as random graphs, complex network theory. Therefore, this article will be based GIS and geospatial network to discuss urban street network. Bus network topology characteristics were analysis, Fig. 2 shows the relationship between the number of connections and the cumulative probability between transit network, and Fig. 3 for double logarithmic graph.

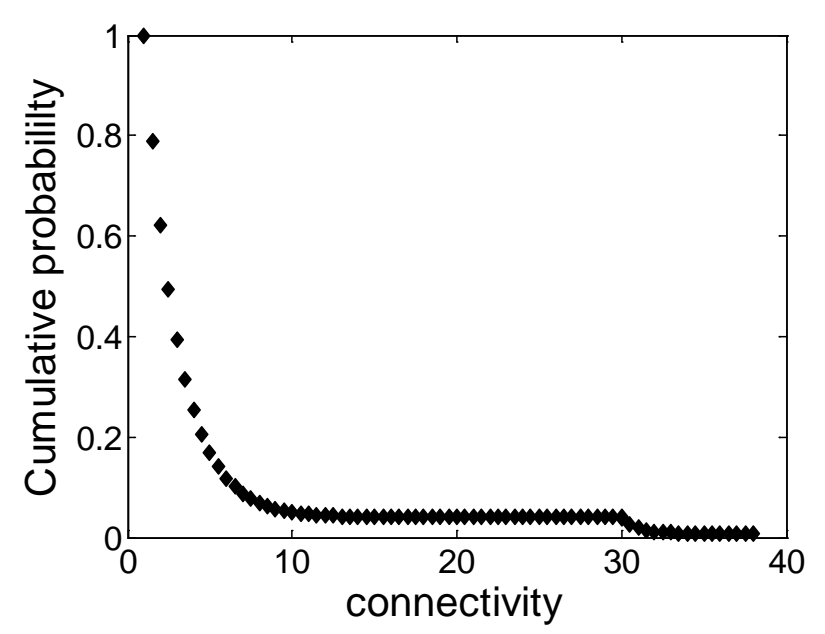

Figure 2. The relationship between the numbers of connections

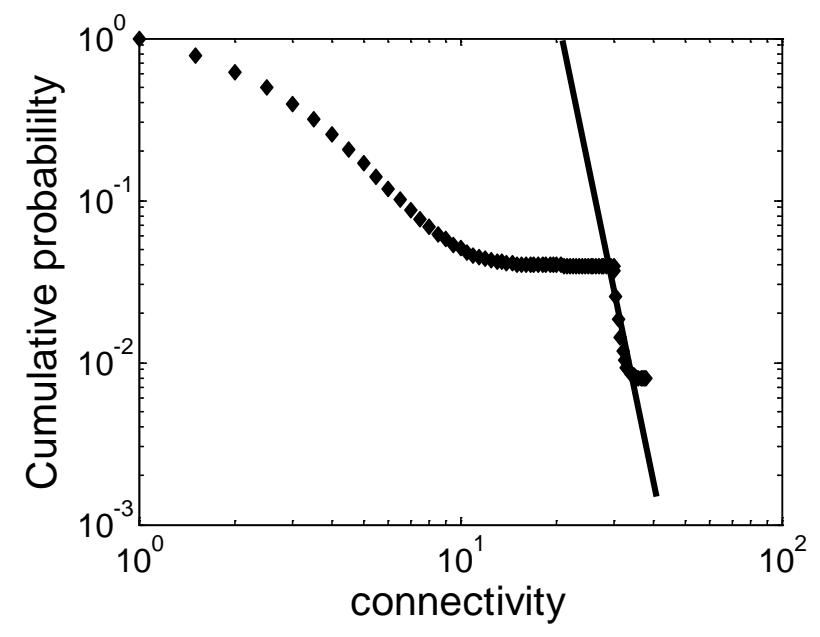

Figure 3. Double logarithmic graph 
City street network is the basis of socio-economic and physical facilities of people's lives, the nature of urban road network design and pattern for better planning of urban roads, pedestrian and traffic flow study law and choose the location of commercial outlets have considerable significance. Study the topological properties of the urban road network. Combining GIS and complex networks. Although our study is each city developed under different historical! Economic and cultural conditions, but show surprisingly similar pattern, and random networks, but also improve the planning and advance through different cities.

Unlike other network traffic is characterized by the weighted complex networks, network traffic is social and economic networks, which is a stream of people, have the ability to judge selection, selection of the optimal route based on his economic costs. Therefore, in considering the overall network, consider the reaction of the crow. People's choice will affect the overall performance of the transport network. Traffic areas of scientific research that the transport infrastructure network traffic is to give travelers a service, the service level of traffic and traffic is relevant. Consider the choice of travel routes, and the system of collective choice because travelers' presents different states, which in turn affects the travelers' travel time and money.

\section{Summary}

This paper follows the general understanding of things research ideas, according to research from the empirical modeling of theoretical priorities, focus on the characteristics of the transport network, progressive approach to the complex nature of the traffic on the topology of the network, in-depth study of the internal mechanism of the system. Traveler discovery and network traffic based on cost related to the options, and then reverse impact study topological properties of the underlying transport network for travel behavior on transport networks, evaluate proposed transport network efficiency indicators. Finally, the transport network services to provide transport services consisting of transportation supply chain research, describes the future of transportation and supply chain research content.

\section{References}

[1] Yao Meng, Tiejun Zhao, Sheng Li, Jianming Yao, " Based on the evaluation of the English syntactic structure disambiguation and self evaluation rule correction", Journal of Computer Research and Development, vol. 39, no. 7, pp. 802-808, 2002.

[2] He Yan Huang, Zhao Xiong Chen, "Based on analysis of complex long sentence translation algorithm ", Journal of Chinese information processing, vol. 16, no. 3, pp. 1-2, 2002.

[3] Li Shutao, Wang Yaonan, Zhang Changfan, "Objective evaluation and analysis of multisensor image fusion", Chinese Journal of Scientific Instrument, vol. 23, no. 6, pp. 651-654, 2002.

[4] WangJue, WangRen, MiaoDuoqian, "Data enriching based on rough set theory", Journal of Environmental Sciences, vol. 29, no. 3, pp. 63-69, 2010.

[5] Zheng Zhonglong, Zhao Jianmin, Yang Jie. "NMF with Log Gabor Wavelets for visualization". Lecture Notesin Computer Scienee, vol. 3691, no. 2, pp. 26-32, 2005.

[6] WangJue, WangRen, MiaoDuoqian, "Data enriching based on rough set theory", Journal of Environmental Sciences, vol. 29, no. 3, pp. 63-69, 2010.

[7] Zhang Xiaodan, H u Feng , Zhao Li, "Improved Shuf F Led Frog Leaping Algorithm Based On Molecular Dynamics Simulations", Journal of Data Acquisition \& Processing, vol.27, no.3, pp.327-332, 2012.

[8] Gg Yu, Wang Xueping, Liang Jing, "Improved Shuffled Frog Leaping Algorithm", Journal of Computer Applications, vol.32, no.1, pp.234-237, 2012.

[9] Rahimi-Vahed A, Mirzaei A H, "A Hybrid Multi-Objective Shuffled Frog-Leaping Algorithm For A Mixed Model Assembly Line Sequencing Problem", Computers and Industrial Engineering, , vol.53,no.4, pp.642-666, 2007.

[10] Zhang X C, Hu X M, Cui G Z, "An Improved Shuffled Frog Leaping Algorithm with Cognitive Behavior ", 7th WCICA Conf. ,pp.6197-6202, 2008. 
[11] Elbctagi E, Hegazy T, Griemon D, "Comparison Among Five Evolutionary-Based Optimization Algorithms", Advanced engineering Informatics, vol.19, no.1, pp.43-53, 2005.

[12] 3GPP TS 36.300, "Evolved Universal Terrestrial Radio Access (E-UTRA) and Evolved UTRA (E-UTRA)”, Dec. 2008. V8.0.0. 